\title{
Hidraulicidad de los silicatos cálcicos
}

\author{
ANA ISABEL TRIVIÑO, Leda. en Farmacia \\ FERNANDO TRIVIÑO, Dr. en Ciencias Químicas \\ CSIC/IETCC
}

\section{$R E S U M E N$}

A pesar de su extensión, este trabajo se puede considerar un breve resumen, pues solamente hemos utilizado una pequeña muestra bibliográfica de la extensisima existente sobre los silicatos cálcicos. Nuestra intención ha sido relacionar las propiedades hidráulicas con las cristalográficas; aclarar las teorias de la estabilización a temperatura ambiente de los compuestos estables a elevadas temperaturas y finalmente describir las posibles causas de la actividad hidráulica de los silicatos. Tema en el que estamos trabajando por lo cual emitimos también ideas propias. También pensamos que hemos hecho una puesta al dia sobre este confuso tema.

\section{$S U M M A R Y$}

In spite of the largeness of this work we can consider it as a brief resumé, since we have employed a little sample among all the wide bibliography existing on calcium silicates. Our aim is to connect the hydraulic properties with the crystallographic ones; to clear up the theories about the stabilization into ambient temperature of the stable composites into high temperatures and finaly to describe the probable reasons of the hydraulic activity of the silicates. We are working in this subject, so, we also expose our own idea and we also think we have put up to date this confused subject.

\section{INTRODUCCION}

El cemento portland es el conglomerante que más se utiliza, hoy dia, en la industria de la construcción. Es la alita o silicato tricálcico su principal componente hidráulico (1) capaz de dar las resistencias mecánicas necesarias para su uso.

El silicato tricálcico tiene dos inconvenientes, igual que tiene dos ventajas; los primeros son: necesita un aporte térmico elevado suministrado por el combustible para su formación y durante su hidratación da hidróxido cálcico que es soluble en aguas puras; las segundas son: da altas resistencias mecánicas y el hidróxido cálcico de hidratación proporciona protección a las armaduras y tubos de hierro sumergidos en el cemento u hormigón.

Las propiedades hidráulicas del $\mathrm{C}_{3} \mathrm{~S}^{*}$ parecen estar relacionadas con una pobre coordinación de los iones calcio que da lugar a la presencia de huecos en la red cristalina (2).

Entre la temperatura ambiente y $\operatorname{los} 1.100^{\circ} \mathrm{C}$ existen 6 formas alotrópicas de silicato tricálcico puro: (3).

$$
\mathrm{T}_{\mathrm{I}} \stackrel{600}{\leftrightharpoons} \mathrm{T}_{\mathrm{II}} \stackrel{920}{\leftrightharpoons} \mathrm{T}_{\mathrm{III}} \stackrel{980}{\leftrightharpoons} \mathrm{M}_{\mathrm{I}} \stackrel{990}{\leftrightharpoons} \mathrm{M}_{\mathrm{II}} \stackrel{1.050}{\leftrightharpoons} \mathrm{R}
$$

$\mathrm{T}=$ Triclinica.

$\mathrm{M}=$ Monoclinica.

$\mathrm{R}=$ Romboédrica.

* En la quimica del cemento se utiliza la siguiente taxonomia: $\mathrm{C}=\mathrm{CaO}: \mathrm{S}=\mathrm{SiO}_{2} ; \mathrm{F}=\mathrm{Fe}_{2} \mathrm{O}_{3}: \mathrm{M}=\mathrm{MgO}: \mathrm{A}=\mathrm{Al}_{2} \mathrm{O}_{3}$. 
Las transformaciones entre los distintos polimorfos del $\mathrm{C}_{3} \mathrm{~S}$ consisten en pequeños desplazamientos atómicos sin rotura de enlaces químicos (4). Esto hace que las transiciones polimórficas del $\mathrm{C}_{3} \mathrm{~S}$ no afecten a las resistencias mecánicas desarrolladas por el cemento después de su hidratación (5).

$\mathrm{La}$ introducción de iones extraños, en el $\mathrm{C}_{3} \mathrm{~S}$, procedentes de otros compuestos presentes en los clinkeres como impurezas: $\mathrm{P}_{2} \mathrm{O}_{5}, \mathrm{SO}_{3}, \mathrm{CaSO}_{4} \cdot 2 \mathrm{H}_{2} \mathrm{O}, \mathrm{CaCl}_{2}, \mathrm{Cr}_{2} \mathrm{O}_{3}$ pueden modificar la reactividad del $\mathrm{C}_{3} \mathrm{~S}$ pero este cambio de reactividad no parece ir asociado con cambios en la estructura (6).

Otro componente con menos proporción de cal del clínker del cemento portland es el silicato bicálcico, que existe en cinco formas polimórficas distintas. Al contrario de lo que ocurre con los polimorfos del $\mathrm{C}_{3} \mathrm{~S}$ el paso de unas formas cristalinas a otras en el $\mathrm{C}_{2} \mathrm{~S}$ supone rotura de enlaces y cambios de agrupaciones iónicas. Esto implica que las propiedades hidráulicas del silicato bicálcico deben variar en función de su estructura cristalina.

Las impurezas presentes en los clinkeres y que pueden estabilizar diferentes formas del $\mathrm{C}_{2} \mathrm{~S}$ pueden, por tanto, afectar también las características hidráulicas del mismo (7).

Desde hace tiempo es sabido que si el silicato tricálcico se sustituyese por el bicálcico, se ahorraría combustible en su fabricación, al tener un mol menos de cal, y se haría un cemento más "durable" por ser menos atacable por aguas puras; pero sus resistencias mecánicas serían más bajas. El objetivo de este trabajo es estudiar las variantes polimórficas del silicato bicálcico en sus formas $\alpha, \alpha^{\prime}$ y $\beta$ y también el efecto de adiciones de otros elementos químicos distintos de la silice y la cal, para aumentar su actividad hidráulica.

Gutt (13) indica que el compuesto $\mathrm{KC}_{23} \mathrm{~S}_{12}$ dado por W.C. Taylor (14) no existe como tal y que el $\mathrm{K}_{2} \mathrm{O}$ con el $\mathrm{C}_{2} \mathrm{~S}$ da, según se temple en agua o al aire, el $\alpha^{\prime} \mathrm{C}_{2} \mathrm{~S}$ o el $\beta \mathrm{C}_{2} \mathrm{~S}$ si se enfría lentamente; también dice que no pudo estabilizar la forma $\alpha \mathrm{C}_{2} \mathrm{~S}$ por adición de $\mathrm{K}_{2} \mathrm{O}$.

Smith Majurdar y Ordway (15) sugieren que las formas $\alpha^{\prime}{ }_{\mathrm{H}}$ y $\alpha_{\mathrm{L}}^{\prime}$ se producen sólo en el enfriamiento del $\mathrm{C}_{2} \mathrm{~S}$ que ha sido calentado antes o previamente a temperaturas del orden de $1.400^{\circ} \mathrm{C}$ y que ha pasado por la forma $\alpha$ de alguna manera. Sin embargo hoy día sabemos que no es necesario llegar a $1.400^{\circ} \mathrm{C}$ para obtener la $\alpha^{\prime} \mathrm{C}_{2} \mathrm{~S}$ ya que se puede obtener a $1.000^{\circ} \mathrm{C}$.

B. Matkowic (16) añade porcentajes crecientes de $\mathrm{Ca}\left(\mathrm{PO}_{4}\right)_{3} \mathrm{OH}$ hasta el $16 \%$, detecta las fases $\alpha$ y $\alpha^{\prime}$, da un gráfico con las relaciones: temperatura, $\%$ de $\mathrm{P}_{2} \mathrm{O}_{5}$, con un punto de inflexión en $1.100^{\circ}$ y un contenido del 5,5 de $\mathrm{P}_{2} \mathrm{O}_{5}$; para el paso $\alpha_{\mathrm{H}}^{\prime}$ a $\alpha \mathrm{C}_{2} \mathrm{~S}$ indica asimismo como estabilizador al $\mathrm{BaSO}_{4}$; con contenidos del $15 \%$ identificó por DRX como una mezcla de $\beta, \alpha$ y $\alpha^{\prime}$, sin dar los datos de identificación de DRX para cada uno de los polimorfos.

Trömel y Möller (17) dan el difractograma de rayos X tomado en película en un difractómetro de alta temperatura, pero no dan los espaciados.

Sin embargo Nurse (8) da los espaciados de la Nagelschimidtita $\alpha \mathrm{C}_{2} \mathrm{~S}$, de la Bredigita que dice ser $\alpha^{\prime} \mathrm{C}_{2} \mathrm{~S}$, de la Larnita $\beta \mathrm{C}_{2} \mathrm{~S}$ y del $\gamma \mathrm{C}_{2} \mathrm{~S}$ y dice que es imposible obtener puras, sin estabilizadores, las formas $\alpha, \alpha^{\prime}$ y $\beta$.

Los espaciados de la Nagelschimidtita no coincide con los datos, posteriormente por Yamaguchi (18), para la forma $\alpha$ de alta temperatura, obtenidos en difractómetro de alta temperatura y hoy dia se obtiene sin estabilizadores la forma $\beta$.

Alguna de las lineas de la Bredigita tampoco coinciden con las del $\alpha^{\prime}$ de alta temperatura dadas posteriormente por Regourd (19) o Yamaguchi (18). 
Las de la Lárnita tampoco coinciden con las de $\beta \mathrm{C}_{2} \mathrm{~S}$ dadas por Yannakis (20) y las de $\gamma \mathrm{C}_{2} \mathrm{~S}$, con ligeras diferencias, coinciden con las dadas por Midgley (21) en 1974. Lo anterior es explicado previsoriamente por Nurse que dice que: no son lo mismo los resultados obtenidos a elevadas temperaturas (por los efectos de expansión térmica, sobre el difractómetro y sobre la red del cristal estudiado); que los obtenidos de diversos compuestos estabilizados por adiciones ajenas a los silicatos, aunque en algunos casos esto no es asi y también depende de la distorsión de la posición de las líneas de difracción ocasionadas por fuertes adiciones, o por formarse compuestos diferentes de los que se pretendian conseguir.

Por otra parte, nosotros creemos que las cámaras de película de Debije Scherrer normales en la década 40-50 dan distintos fondos, a veces muy altos, que interfieren la interpretación de las lineas débiles. Tampoco tienen el mismo poder separador de lineas que las cámaras con monocromadores de Guinier o los difractómetros, más utilizados hoy día.

Lo anterior es fácilmente comprobable observando las fichas antiguas realizadas sobre datos de pelicula de cámaras Debije Scherrer A.S.T.M. (22) comparando sus resultados con los obtenidos con un difractómetro. Por otra parte el deseo de estabilizar las formas de alta temperatura por adiciones, en algunos casos muy elevadas, o el intentar buscar compuestos en la naturaleza estables pretendidamente semejantes a los polimorfos de alta temperatura; ha ocasionado numerosas confusiones y discusiones que aún se mantienen hoy día.

El que la forma $\alpha$ admita para estabilizarse compuestos extraños puede ser la causa de su pretendida mayor actividad hidráulica. Parece ser que la actividad hidráulica es más dependiente de las adiciones estabilizantes que del polimorfo dado, salvo para la forma $\gamma$ que al no necesitar adiciones estabilizantes, posiblemente no es hidráulica, por ser la foma del silicato bicálcico más pura.

Hoy dia sabemos que existen cinco formas cristalinas del $\mathrm{C}_{2} \mathrm{~S}$ entre la temperatura ambiente y $1.500^{\circ} \mathrm{C}$. Los márgenes de estabilidad de estas formas son distintos según que la variación de temperatura sea en sentido creciente o decreciente.

Niesel y Thormamm (23) representan esquemáticamente los rangos de estabilidad de los diferentes polimorfos del silicato bicálcico de la siguiente manera:

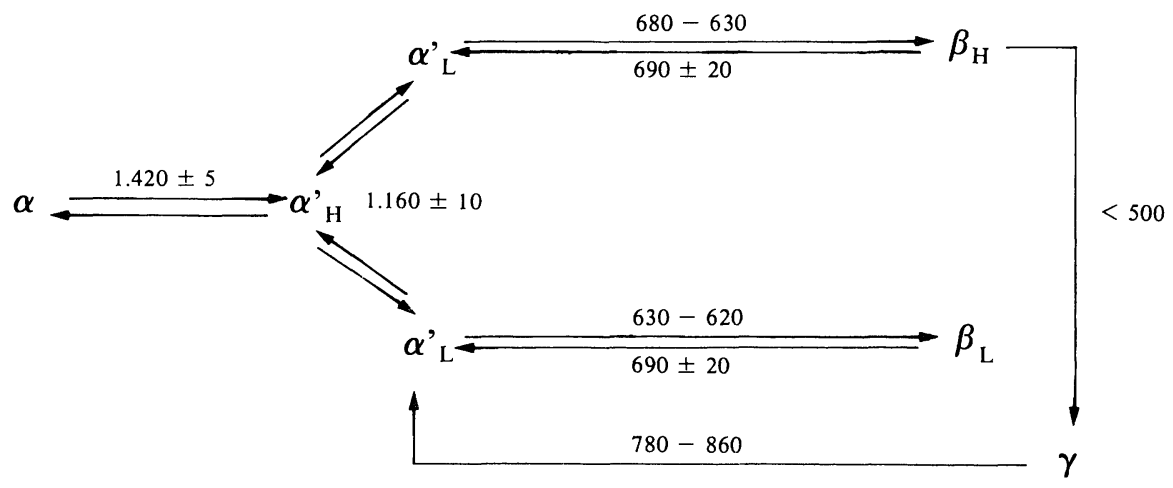

\section{ESTUDIO CRISTALOGRAFICO DE LOS POLIMORFOS DEL SILICATO BICALCALCICO}

Las estructuras cristalinas de estos polimorfos están construidas a base de tetraedros de $\mathrm{SiO}_{4}$ independientes y unidos por átomos de calcio, variando la disposición espacial de estas estructuras de unas formas a otras.

La forma Y (Gamma) presenta una red cristalina ortorrómbica tipo olivino (24). 
La forma $\mathrm{B}$ (beta) es monoclínica con una estructura tipo $\mathrm{K}_{2} \mathrm{SO}_{4}$ deformada (25).

Las formas $\alpha_{\mathrm{L}}^{\prime}$ (alfa prima baja) y $\dot{\alpha}_{\mathrm{H}}$ (alfa prima alta) son ortorrómbicas $(19,26)$ y la forma $\alpha$ (alfa) es trigonal (27).

\section{1. $\alpha-\mathrm{C}_{2} \mathrm{~S}$ :}

Yamaguchi, Ono, Kawamura y Soda (28) y Bredig (27) por otra parte determinaron las dimensiones de la celda unidad de la forma $\alpha$ del silicato bicálcico obteniendo como resultados concordantes ( $\mathrm{a}=5,45 \AA ; \mathrm{c}=7,19 \AA)$.

Estas determinaciones fueron realizadas sobre los resultados obtenidos mediante difractometría de rayos $\mathrm{X}$ en horno de alta temperatura a $1.500^{\circ} \mathrm{C}$.

Sin embargo, las diferencias de resultados obtenidos sobre $\alpha-\mathrm{C}_{2} \mathrm{~S}$ estabilizados a temperatura ambiente mediante adición de distintos compuestos químicos, no coinciden con los resultados anteriores (28); esto puede explicarse porque la necesaria adición de grandes proporciones de agentes estabilizadores puede alterar las dimensiones de la red.

3.2. $\alpha^{\prime}-\mathrm{C}_{2} \mathrm{~S}$ : Existen dos formas de $\alpha^{\prime}-\mathrm{C}_{2} \mathrm{~S}: \alpha_{\mathrm{H}}^{\prime}, \alpha_{\mathrm{L}}^{\prime}(9,16)$.

La transformación de una estructura en otra tiene lugar sin rotura de ningún enlace de coordinación primario y ha sido detectada por micro-DTA, difracción de rayos X, microscopía $\mathrm{y}$ dilatometria (29). La diferencia entre una estructura y otra es debida sólo a un desplazamiento de los átomos de calcio.

La transformación $\alpha \rightarrow \alpha^{\prime}$, observada claramente por DTA y difracción de rayos X, se caracteriza por una rotación de los tetraedros de silice.

Smith, Majumdar y Ordway (24) obtuvieron líneas de difracción de $\alpha^{\prime}{ }_{\mathrm{L}} \mathrm{C}_{2} \mathrm{~S}$ distintas según que esta forma se consiguiera calentando $\mathrm{Y}_{-} \mathrm{C}_{2} \mathrm{~S}$ o por enfriamiento a partir de la forma $\alpha$.

Yamaguchi, Ono, Kawamura y Soda (28) encontraron los siguientes parámetros para una forma $\alpha$ estabilizada por $10 \% \mathrm{CaMgSiO}_{4}$ y $4 \% \mathrm{~K}_{2} \mathrm{O} ; \mathrm{a}=6,748, \mathrm{~b}=5,494, \mathrm{c}=9,261 \AA$. Esta forma corresponde a un $\alpha^{\prime}{ }_{\mathrm{H}} \mathrm{C}_{2} \mathrm{~S}$ cuyos parámetros determinados a alta temperatura por los mismos autores son: $\mathrm{a}=6,883 ; \mathrm{b}=5,600 ; \mathrm{c}=9,543 \AA$.

No obstante, Regourd y Guinier (29) afirman que la forma $\alpha^{\prime}{ }_{\mathrm{H}}$ no puede ser obtenida a temperatura ambiente.

\section{3. $\quad \mathrm{B}-\mathrm{C}_{2} \mathrm{~S}$}

Yannaquis, utilizando una preparación sintética de $\mathrm{B}-\mathrm{C}_{2} \mathrm{~S}$ puro $(20)$ determinó los parámetros de la celda unidad del $\mathrm{B}_{-} \mathrm{C}_{2} \mathrm{~S}: \mathrm{a}=5,507 ; \mathrm{b}=6,754 ; \mathrm{c}=9,317 \AA$, dimensiones que corresponden al sistema monoclínico.

Estos resultados coinciden con los de Yamaguchi, Ono, Kawamura y Soda (28).

Otras determinaciones realizadas sobre $\mathrm{B}-\mathrm{C}_{2} \mathrm{~S}$ estabilizado a temperatura ambiente por adición de ciertos compuestos proporcionan unos valores de estos parámetros algo distintos de los anteriores, lo cual es explicable, como se mencionaba anteriormente, por las distensiones producidas por los iones extraños introducidos. 


\section{4. $\gamma-\mathrm{C}_{2} \mathrm{~S}$}

Es la forma estable a temperatura ambiente. Los parámetros de la celda unidad han sido determinados por diversos autores y sus resultados son concordantes. Estos parámetros son: $\mathrm{a}=5,076 ; \mathrm{b}=6,756 ; \mathrm{c}=11,230 \AA$.

La transformación $\mathrm{B} \rightarrow \mathrm{Y}$ lleva consigo una expansión de volumen que determina que los cristales estallen en fragmentos muy pequeños.

Como es sabido esta forma no es hidráulica.

\section{ESTABILIZACION DE LOS DIFERENTES POLIMORFOS DEL SILICATO BICALCICO}

Como ya hemos dicho, a temperatura ambiente la forma estable del $\mathrm{C}_{2} \mathrm{~S}$ es la forma $\mathrm{Y}$, sin embargo las demás formas, estables a más altas temperaturas pueden ser obtenidas también a temperatura ambiente.

\subsection{Estabilización de $B-\mathrm{C}_{2} \mathrm{~S}$ :}

La forma $\mathrm{B}$, es la única forma del silicato bicálcico estable a alta temperatura que ha podido obtenerse pura a temperatura ambiente. Las variables que pueden influir evitando la transformación $\mathrm{B} \rightarrow \mathrm{Y}$ son las siguientes:

a) Tamaño de los cristales (30): la reducción del tamaño de los cristales de la forma alfa y alfa prima debajo de 10 o mejor 5 micras de diámetro favorece la estabilización de la forma a temperatura ambiente. Este efecto puede ser explicado por el mecanismo de la transformación $\beta \rightarrow \gamma$ la cual tiene lugar por nucleación y aumento de volumen.

En el rango de estabilidad de la fase $\alpha$, la razón velocidad de crecimiento/velocidad de nucleación, es baja o casi nula. Según Niesel y Thormann (23) $1.160^{\circ} \mathrm{C}$ sería la temperatura critica de crecimiento para los cristales de $\alpha^{\prime}-\mathrm{C}_{2} \mathrm{~S}$; si al $\mathrm{C}_{2} \mathrm{~S}$ se sintetiza por debajo de $1.160^{\circ} \mathrm{C}$ se podría obtener $\mathrm{B}$, mientras que una muestra calentada por encima de $1.160^{\circ} \mathrm{C}$ daría una mezcla B y Y al enfriar a temperatura ambiente. Nuestros resultados no son concordantes al $100 \%$ con esta afirmación, ya que el máximo de $\mathrm{B}_{-} \mathrm{C}_{2} \mathrm{~S}$ se encuentra en muestras calentadas a $1.220^{\circ} \mathrm{C}$ y el de $\mathrm{Y}-\mathrm{C}_{2} \mathrm{~S}$ en muestras templadas desde $1.434^{\circ} \mathrm{C}$ lo cual implica que el máximo de nucleación está próximo al punto de transformación $\alpha^{\prime} \rightarrow \alpha$.

Por ejemplo según A. A. Pashochenko (34) a $1.000^{\circ} \mathrm{C}$ las partículas de la forma beta tienen un tamaño de $0,02 \mathrm{~mm}$, a $1.350^{\circ} \mathrm{C}$ crecen hasta $0,25 \mathrm{~mm}$ y aparecen estrias a dicha temperatura. Las muestras calentadas a temperaturas inferiores a $1.100^{\circ} \mathrm{C}$ no dan la forma gamma. Las muestras calentadas a más de $1.170^{\circ} \mathrm{C}$ se pulverizan. Las belitas sintetizadas a $1.000^{\circ} \mathrm{C}$ dan las máximas resistencias mecánicas.

$\begin{array}{cccccccc}{ }^{\circ} \mathrm{C} & 700 & 800 & 900 & 1.000 & 1.100 & 1.200 & 1.300 \\ \mathrm{kp} / \mathrm{cm}^{2} & 240 & 375 & 450 & 750 & 500 & 475 & 200 \\ 28 \text { dias } & & & & & & \end{array}$

Nucleación. Si calentamos la forma alfa a $1.550^{\circ} \mathrm{C}$ da un $90 \%$ de beta si se templa, debido a que el paso alfa, alfa prima es rápido y los cristales permanecen pequeños. Si se enfría lentamente de $1.550^{\circ} \mathrm{C}$ a $1.400^{\circ} \mathrm{C}$ y se templa desde $1.400^{\circ} \mathrm{C}$ hay un $95 \%$ de Y. Por encima de $1.160^{\circ} \mathrm{C}$ se obtiene $\mathrm{Y}$ por debajo $\mathrm{B}$. Añadiendo un $1 \%$ de cal se encuentran cristales $0,01 \mathrm{~mm}$ de la forma beta. 
b) Presión: según Roy (32) la fase $\mathrm{B}$ es estable a temperatura ambiente a 2 atmósferas de presión.

c) Mezclas no estequiométricas: La formación de soluciones sólidas de $\mathrm{CaO}$ en el silicato dicálcico promueve la estabilización de la forma B (33). En nuestro caso encontramos que la forma estabilizada por un exceso de cal es más bien la gamma.

d) Temperatura de calentamiento: Como ya se ha dicho, según Niesel y Thormann puede obtenerse $\mathrm{B}-\mathrm{C}_{2} \mathrm{~S}$ calentando por debajo de $1.160^{\circ} \mathrm{C}$. Sin embargo Korveev y Bygalinc (33) afirman que calentando repetidamente en el rango de $900-1.500^{\circ} \mathrm{C}$ se puede obtener $\mathrm{B}-\mathrm{C}_{2} \mathrm{~S}$ puro. Esto último no concuerda con nuestros resultados.

Pritts y Daugherty (34) han obtenido $\mathrm{B}-\mathrm{C}_{2} \mathrm{~S}$ puro calentando a $1.100^{\circ} \mathrm{C}$ y enfriando lentamente, siendo necesario largos tiempos de calentamiento repetidas veces para reducir el contenido de cal libre por debajo del $0,1 \%$.

En nuestra opinión el suministrador de cal debe de reaccionar a la temperatura más baja posible, de esta forma lo que es difícil de conseguir con el $\mathrm{CaCO}_{3}$, es más fácil de realizar con el $\mathrm{Ca}(\mathrm{OH})_{2}, \mathrm{CaCl}_{2}, \mathrm{Ca}\left(\mathrm{NO}_{3}\right)_{2}$ de menores temperaturas de descomposición en óxidos.

Smith J. (24) dice que el paso a gamma sucede en todas las muestras calentadas durante 15 minutos y templadas de 1.430 a $1.500^{\circ} \mathrm{C}$. Lo anterior no sucede en las calentadas a $1.410^{\circ} \mathrm{C}$ en las que aparece mezcla de beta a gamma; enfriando lentamente pasan a gamma en su totalidad. $\mathrm{Al}$ añadir $\mathrm{Al}_{2} \mathrm{O}_{3}$ la temperatura crítica de inversión se encontró entre $1.370-1.380^{\circ} \mathrm{C}$.

M. Gawlicki (35) observa que la temperatura de pulverización por paso $(\mathrm{B} \rightarrow \mathrm{Y})$ sube al aumentar el tiempo de recocido de 10 minutos a 180 minutos y también al aumentar las temperaturas de $1.200^{\circ} \mathrm{C}$ a $1.500^{\circ} \mathrm{C}$. El \% de forma gamma crece también encima de $1.450^{\circ} \mathrm{C}$.

Con un contenido de gamma del 40 al $47 \%$ como máximo no hay autopulverización esto contribuye a muchos errores de interpretación que confunden el que no haya autopulverización con no existencia de la fase gamma.

Una de las formas de obtener $\mathrm{B}-\mathrm{C}_{2} \mathrm{~S}$ a temperatura ambiente es mediante la adición de estabilizadores.

Suzuki y Tsujita (37) han comprobado que los cationes $\mathrm{Cr}^{3+}, \mathrm{V}^{5+}, \mathrm{P}^{5+}$ y $\mathrm{B}^{3+}$ estabilizan la forma $\mathrm{B}$ en una concentración entre $2 \%$ y $8 \%$. Por encima de esta concentración se obtiene también forma $\alpha$. Sólo el $\mathrm{B}^{3+}$ estabiliza $\alpha^{\prime}$ en lugar de $\alpha$.

En nuestro caso la estabilización es de $\beta \rightarrow \alpha^{\prime} \rightarrow \gamma$.

El Al y $\mathrm{Fe}$ también eran capaces de estabilizar $\mathrm{B}-\mathrm{C}_{2} \mathrm{~S}$ pero enfriando la muestra rápidamente en agua, sin embargo el $\mathrm{Mn}^{2+}$ no estabiliza la forma $\mathrm{B}$, la cual se obtiene siempre mezclada con Y.

El $\mathrm{B}_{2} \mathrm{O}_{3}$ es el estabilizador clásico para el $\mathrm{B}-\mathrm{C}_{2} \mathrm{~S}$.

Usando una concentración menor que el $0,5 \%$ de $\mathrm{B}_{2} \mathrm{O}_{3}$ la red cristalina del $\mathrm{B}_{-} \mathrm{C}_{2} \mathrm{~S}$ no se altera apreciablemente.

Los óxidos de $\mathrm{Na}_{2} \mathrm{O}$ y $\mathrm{K}_{2} \mathrm{O}$ también estabilizan la forma $\mathrm{B}$ según algunos autores (38). 
Suzuki (39) ha obtenido $\mathrm{B}-\mathrm{C}_{2} \mathrm{~S}$ estable a temperatura ambiente y con la siguiente composición en'moles \%: $\mathrm{Ca}_{1,85}, \mathrm{Na}_{0,15}, \mathrm{Si}_{0,90}, \mathrm{Fe}_{0,10}, \mathrm{O}_{4}$ o bien $\mathrm{Ca}_{1,85}, \mathrm{~K}_{0,15}, \mathrm{Si}_{0,90}, \mathrm{Fe}_{0,10} \mathrm{O}_{4}$. se obtenía forma $\mathrm{B}$ cuando el $\mathrm{Fe}$ era sustituido por $\mathrm{Al}$. Las temperaturas a las que fueron calentadas las muestras fueron $1.520^{\circ} \mathrm{C}$ y $1.440^{\circ} \mathrm{C}$

\subsection{Estabilización de $\alpha^{\prime}-\mathrm{C}_{2} \mathrm{~S}$}

Suzuki y Yamaguchi (26) han obtenido $\alpha^{\prime}-\mathrm{C}_{2} \mathrm{~S}$ a temperatura ambiente sustituyendo el $\mathrm{CaO}$ en el $\mathrm{C}_{2} \mathrm{~S}$ por $\mathrm{BaO}$ o $\mathrm{SrO}$ y también por sustitución del $\mathrm{SiO}_{2}$ por $\mathrm{B}$. Cuando sustituian $0,15-0,30$ moles de $\mathrm{CaO}$ por $\mathrm{BaO}$ o $\mathrm{SrO}$ y calentaban $20^{\prime}$ a $1.500^{\circ} \mathrm{C}$ obtenían cristales de $\alpha^{\prime}-\mathrm{C}_{2} \mathrm{~S}$ de relativamente gran tamaño. La composición de estos cristales era $56,12 \% \mathrm{CaO}, 11,21 \% \mathrm{BaO}$ y $32,67 \% \mathrm{SiO}_{2}$ o bien $57,84 \% \mathrm{CaO}, 8,65 \% \mathrm{SrO}$ y $33,51 \% \mathrm{SiO}_{2}$.

Cuando sustituian el Si por B, la composición de los cristales $\alpha^{\prime}$ que obtenían era: $67,88 \%$ $\mathrm{CaO}, 31,17 \% \mathrm{SiO}_{2}, 0,95 \% \mathrm{~B}_{2} \mathrm{O}_{3}$, después de calentar a $1.450-1500^{\circ} \mathrm{C}$.

Asimismo, Suzuki y Tsujita ha obtenido $\alpha^{\prime}-\mathrm{C}_{2} \mathrm{~S}$ mediante la adición de $\mathrm{B}_{2} \mathrm{O}_{3}(12-20$ moles \%) y calentando a $1.500^{\circ} \mathrm{C}(39)$.

Con $\mathrm{Na}-\mathrm{Al}, \mathrm{Na}-\mathrm{Fe}, \mathrm{K}-\mathrm{Al}$ y $\mathrm{K}-\mathrm{Fe}$ siempre se obtenía una mezcla de $\alpha^{\prime}-\mathrm{C}_{2} \mathrm{~S}$ con $\mathrm{B}_{-} \mathrm{C}_{2} \mathrm{~S}$ o $\alpha-C_{2} \mathrm{~S}$ dependiendo de la concentración de los respectivos óxidos que se añadiera y de la temperatura de calentamiento.

Esos mismos autores obtuvieron $\alpha^{\prime}-\mathrm{C}_{2} \mathrm{~S}$ estabilizado con $\mathrm{MgO}$ y con la siguiente composición quimica: $1,8 \mathrm{CaO}, 0,2 \mathrm{MgO}, 1 \mathrm{SiO}_{2}$.

Ghosh (40) describe la preparación de $\alpha^{\prime}-\mathrm{C}_{2}$ estable a temperatura ambiente en presencia de 1-3\% de $\mathrm{B}_{2} \mathrm{O}_{3}$. Asimismo obtenía $\alpha^{\prime}-\mathrm{C}_{2} \mathrm{~S}$, junto con $\mathrm{B}^{-} \mathrm{C}_{2} \mathrm{~S}$, en presencia de $\mathrm{NaF}$. La fase $\alpha^{\prime}$ desaparecia por adición de yeso $\left(\mathrm{CaSO}_{4}\right)$ o $\mathrm{CaS}$.

Udagawa y Urabe (41) afirman que la mezcla estequiométrica de $\mathrm{CaCO}_{3}, \mathrm{SrCO}_{3}$ y $\mathrm{SiO}_{2}$ correspondiente a la fórmula $\left(\mathrm{Ca}_{1,7} \mathrm{Sr}_{0,3}\right) \mathrm{SiO}_{4}$ calentada a $1.500^{\circ} \mathrm{C}$ durante dos horas, permitía obtener cristales de $\alpha^{\prime}-\mathrm{C}_{2} \mathrm{~S}$ cuyos parámetros reticulares corresponden a la fase $\alpha^{\prime}$ estable a temperatura más baja: $\alpha^{\prime}{ }_{\mathrm{L}}-\mathrm{C}_{2} \mathrm{~S}$.

Regourd (29) estudiando los parámetros reticulares de las diferentes modificaciones del $\mathrm{Ca}_{2} \mathrm{SiO}_{4}$ afirma que mediante la adición de $4,3 \% \mathrm{~K}_{2} \mathrm{O}$ o de $8,65 \% \mathrm{SrO}$ al $\mathrm{C}_{2} \mathrm{~S}$ obtiene cristales cuyas constantes de red corresponden a las del $\alpha_{\mathrm{L}}^{\prime}-\mathrm{C}_{2} \mathrm{~S}(\mathrm{a}=11,02 ; \mathrm{b}=18,69 ; \mathrm{c}=6,83 \AA)$.

\subsection{Estabilizantes de $\alpha-\mathrm{C}_{2} \mathrm{~S}$ :}

La fase $\alpha$ del silicato bicálcico puede ser sintetizada fácilmente a temperatura ambiente utilizando como estabilizadores $\mathrm{Na}$ y $\mathrm{Fe}$ o $\mathrm{K}$ y $\mathrm{Fe}$ (39).

La composición de las mezclas estequiométricas fue la necesaria para dar:

$\mathrm{Ca}_{1,75}, \mathrm{Na}_{0,25}, \mathrm{Si}_{0,75}, \mathrm{Fe}_{0,25} \mathrm{O}_{4}$ o bien $\mathrm{Ca}_{1,75}, \mathrm{~K}_{0,25}, \mathrm{Si}_{0,75}, \mathrm{Fe}_{0,25} \mathrm{O}_{4}$ y las temperaturas de calentamiento fueron $1.520^{\circ} \mathrm{C}, 1.440^{\circ} \mathrm{C}$ y $1.360^{\circ} \mathrm{C}$.

Asimismo estos autores han comprobado que cuando los clínkeres se mantienen suficiente tiempo a alta temperatura y son enfriados rápidamente desde cerca del limite más bajo de temperatura a que es estable el $\alpha-\mathrm{C}_{2} \mathrm{~S}$, la cantidad de fase $\alpha$ en la belita del clínker incrementa. 
Cuando los clinkeres son enfriados lentamente, el $\alpha-\mathrm{C}_{2} \mathrm{~S}$ revierte completamente a $\alpha^{\prime}-\mathrm{C}_{2} \mathrm{~S}$.

Suzuki y Yamaguchi (26) han estabilizado la forma $\alpha$ sustituyendo 0,7 moles de $\mathrm{CaO}$ por $\mathrm{BaO}$.

Sin embargo, la sustitución de 0,7 moles de $\mathrm{CaO}$ por SrO producía sólo $\alpha$ '.

Suzuki y Tsujita (27) han conseguido estabilizar también la forma $\alpha$ mediante la adición de 18$20 \%$ de $\mathrm{Cr}_{2} \mathrm{O}_{3}$ y calentando a $1.500^{\circ} \mathrm{C}$. Con un 24-30\% de $\mathrm{V}_{2} \mathrm{O}_{5}$ también obtuvieron $\alpha-\mathrm{C}_{2} \mathrm{~S}$ a temperatura ambiente, así como con un $20-26 \%$ de $\mathrm{P}_{2} \mathrm{O}_{5}$.

Nurse R. W. (42) opina que durante la inversión alfa prima, alfa; hay un aumento de volumen y que es probable que la forma alfa disuelva cantidades apreciables de impurezas. Lo anterior se daría para el fósforo en la forma $9 \mathrm{CaO}_{2} \mathrm{O}_{5} 3 \mathrm{SiO}_{2}$. La forma alfa- $\mathrm{C}_{2} \mathrm{~S}$ puede llegar a tener en disolución $3,6 \% \mathrm{CaO}, 2,8 \% \mathrm{Na}_{2} \mathrm{O}, 3,8 \% \mathrm{de}_{\mathrm{Al}_{2} \mathrm{O}_{3}}$ con un $89,8 \%$ de $\mathrm{C}_{2} \mathrm{~S}$.

El $\mathrm{Na}$ y $\mathrm{Ca}$ en exceso neutralizan el efecto negativo del $\mathrm{AlO}_{4}$ en el caso anterior.

Cuando un óxido ácido como el $\mathrm{B}_{2} \mathrm{O}_{3}$ se añade al $\mathrm{C}_{2} \mathrm{~S}$ en el sistema $\mathrm{CaO}-\mathrm{B}_{2} \mathrm{O}_{3}-\mathrm{SiO}_{2}$ las disoluciones sólidas son todas más ricas en $\mathrm{CaO}$ que las composiciones situadas entre $\mathrm{C}_{2} \mathrm{~S}$ y $\mathrm{B}_{2} \mathrm{O}_{3}$, parte del $\mathrm{CaO}$ entra al $\mathrm{C}_{2} \mathrm{~S}$ para formar $\mathrm{C}_{3} \mathrm{~S}_{2}$. En los experimentos de Newman el efecto del estabilizador ácido fue un cambio, en la temperatura de inversión de la forma rica en cal, a su valor normal.

En el microscopio la forma alfa se observa poligonal, la alfa prima poligonal estriada y rayada y la beta redondeada y rayada.

Resumiendo existen las siguientes teorias:

1. La forma alfa admite sustancias disueltas que bajan el punto de cambio alfa, alfa prima y que no pueden insolubilizarse o separarse por haber bajado el punto de inversión es decir son retenidas.

2. Ciertas sustituciones en la red del silicato bicálcico alargan el eje $\mathrm{C}$ exagonal y estabilizan la forma alfa.

3. El reemplazamiento del grupo $\mathrm{SiO}_{4}^{4}$ por grupos menores como el $\mathrm{BO}_{4}^{5-}$ estabiliza la forma beta por el exceso de carga requerida para la entrada de iones $\mathrm{Ca}$ o similares en posiciones intersticiales.

4. El reemplazamiento de grupos $\mathrm{SiO}_{4}^{4}$ por grupos de carga menores como el $\mathrm{PO}_{4}^{3-}$ tiene menor efectividad que el caso anterior para eliminar el $\mathrm{Ca}^{++}$de sus posiciones normales.

5. El efecto de la presión y velocidad de nucleación debe de considerarse del tipo de estabilización de la cristaloquímica.

Gren (10) dice que los álcalis, alúmina y $\mathrm{Fe}_{2} \mathrm{O}_{3}$ bajan el punto de inversión alfa-alfa prima cientos de grados centigrados.

Guinier, Smith, Majundar y Ordway (24) dicen que en el cambio alfa, alfa prima hay un giro de medio tetraedro de $\mathrm{SiO}_{2}$, ya que sus vértices apuntan en direcciones opuestas al cambio de fase. La temperatura, al aumentar, facilita la transformación cuando el cambio de fase requiere una reestructuración. 


\section{Teorias sobre actividad hidráulica}

Como se mencionaba al principio de la introducción, las propiedades hidráulicas del $\mathrm{C}_{3} \mathrm{~S}$ parecen estar asociadas a la pobre coordinación de los iones $\mathrm{Ca}^{2+}$ y a la presencia de huecos en la red cristalina.

La forma $\gamma$ del siilcato bicálcico es inerte o muy débilmente hidráulica, lo cual puede ser explicado por la coordinación simétrica de los iones $\mathrm{Ca}^{2+}$ y por la fuerza de los enlaces $\mathrm{Ca}-\mathrm{O}$ consecuencia de la baja coordinación de los iones $\mathrm{Ca}^{2+}(24)$.

En la forma $\beta$, la coordinación poliédrica de los iones $\mathrm{Ca}^{2+}$ es irregular y los enlaces $\mathrm{Ca}-\mathrm{O}$ son más largos facilitando la hidratación.

Pritts y Dangherty (34) han comprobado que la hidratación de la forma B estabilizada por distintos iones crece, en proporción inversa a como lo hace la relación carga/radio de dichos iones. Ejemplo: \% hidratación: $\mathrm{V}^{5+}>\mathrm{Cr}^{6+}>\mathrm{B}^{3+}>\mathrm{S}^{6+}$ siendo la relación carga/radio: $\mathrm{S}^{6+}>\mathrm{B}^{3+}>\mathrm{Cr}^{6+}>\mathrm{V}^{5+}$

Estos autores han observado que los enlaces $\mathrm{Si}-\mathrm{O}$ y $\mathrm{Ca}-\mathrm{O}$ ó $\mathrm{Me}-\mathrm{O}$ en caso de la sustitución tanto del calcio como del silicato por un metal se rompen durante el proceso de hidratación. Por tanto, cuanto mayor sea la relación carga/radio del ión que sustituya al $\mathrm{CaO}$ al $\mathrm{Si}$ en la red cristalina, más fuertes serán los enlaces con el oxígeno y, por tanto, más difícil será la hidratación.

$\mathrm{Al}$ aumentar la concentración del metal también aumenta la fortaleza del enlace $\mathrm{M}-\mathrm{O}$ y por lo tanto también disminuye la velocidad de hidratación.

Cuando las sustituciones son de metales por el calcio los efectos son aún más fuertes, ya que una relación $\mathrm{C} / \mathrm{R}$ para el potasio $\mathrm{K}=0,8$ es equivalente a la del cromo $\mathrm{Cr}=11,5$; y la del sodio $\mathrm{C} / \mathrm{R}=1$ da una velocidad de hidratación semejante a la del $\mathrm{SO}_{3}$ con una $\mathrm{C} / \mathrm{R}=20$. El litio retarda la hidratación, su efecto se explica como ión intersticial, por su actuación sobre la fortaleza de enlace, que provoca una bajada en la temperatura del punto de inversión beta-alfa prima a $714^{\circ} \mathrm{C}$. La teoría de sustitución de anión o catión por $\mathrm{C} / \mathrm{R}$ no explica lo que sucede en el caso del potasio $\mathrm{C} / \mathrm{R}=1,33$ comparado con bario $\mathrm{C} / \mathrm{R}=1,34$ ya que este último no estabiliza la forma beta y tampoco en el caso del vanadio $V^{5}, C / R=0,59$ que estabiliza la forma beta y el aluminio $\mathrm{C} / \mathrm{R}=0,51$ que no la estabiliza.

Entre los factores de tipo físico está el que la introducción de metales provoca una disminución de la rugosidad superficial externa y también la disminución de fisuras que causan una bajada en la actividad superficial y por lo tanto de la velocidad de hidratación.

Si el metal sustituyente tiene mayor radio que el sustituido, cambia la celdilla aumentándola y se favorecen las formas de mayor volumen como la gamma o la alfa.

Como el paso alfa prima-alfa transcurre con un aumento de volumen también es muy posible que la forma alfa tenga mayor poder de disolución de impurezas.

Los fosfatos cálcicos y cálcico sódicos son completamente miscibles con la forma alfa y a baja temperatura tienen estructuras que no son isomorfas con las formas beta y gamma de disolución sólida.

La cantidad de estabilizador disuelto en el $\mathrm{C}_{2} \mathrm{~S}$ influye en la bajada de la temperatura de inversión de fases. Funk y Kantro creen que un aumento de estabilizador disminuye la velocidad de hidratación. 
Pritts dice que la disminución de la temperatura en el paso beta-alfa prima es función del metal disuelto o sustituido en la red y que para el caso del $\mathrm{Cr}_{2} \mathrm{O}_{3}$ puede entrar en la red hasta un $1,8 \%$. Para el $\mathrm{B}_{2} \mathrm{O}_{3}$ la concentración es del orden del $1 \%$. La hidratación del beta silicato bicálcico en una reacción es de primer orden para el periodo segundo de hidratación comprendido entre el llamado inicial durmiente y el lento final de difusión. La energia de activación es 14 para el $\mathrm{Cr}_{2} \mathrm{O}_{3}$ y $12 \mathrm{Kcal} / \mathrm{mol}$ para el $\beta-\mathrm{C}_{2} \mathrm{~S}$ que coinciden con la energía de activación para la polimerización de los silicatos a $\mathrm{pH}$ 10,5; comparando las vélocidades de hidratación éstas disminuyen con aumentos del estabilizador respecto a las del beta $\mathrm{C}_{2} \mathrm{~S}$ puro.

J. Stark y A. Müller (43) dicen que la actividad hidráulica de las belitas depende del grado de desorden de la red cristalina y que las variedades de alta temperatura tienen mayor grado de desorden que las estables a temperatura ambiente, siendo las resistencias mecánicas según ellos $\alpha \mathrm{C}_{2} \mathrm{~S}>\alpha^{\prime} \mathrm{C}_{2} \mathrm{~S}>\beta \mathrm{C}_{2} \mathrm{~S}>\gamma-\mathrm{C}_{2} \mathrm{~S}$; dividen su estudio en tres grupos:

1. Actividad hidráulica de las fases del $\mathrm{C}_{2} \mathrm{~S}$.

2. Actividad hidráulica de cementos beliticos con componentes extraños.

3. Actividad hidráulica de cementos belíticos con componentes añadidos como el $\mathrm{C}_{4} \mathrm{~A}_{3} \mathrm{~S}$.

En el caso 2) Ono Y. (44) dice que por templado enérgico del clínker se mantiene un $40 \%$ de la forma alfa retenida entre la forma beta y no hay exclusión de impurezas.

En el tercer caso la fase belítica inicialmente inerte y contribuye a las resistencias finales, opina que las resistencias mecánicas no pueden ser aumentadas por bajadas del gradiente térmico. La actividad térmica, en la cual la combinación de la cal se acelera por el aumento de la velocidad a que los crudos se calientan a la temperatura de cocido, no está relacionada con las resistencias mecánicas.

La temperatura de cocido no influye en las resistencias mecánicas.

La velocidad de temple para cementos con el $45-60 \%$ de belita con un estándar de cal del 78 al 83 , mejora las resistencias un $300 \%$ por disminución del gradiente térmico de $3.000^{\circ} \mathrm{K} / \mathrm{min}$. con un gradiente térmico de enfriamiento de $100^{\circ} \mathrm{K} / \mathrm{min}$. Enfriando de temperaturas de $1.350^{\circ} \mathrm{C}$ a $900^{\circ} \mathrm{C}$ ya no influye, a temperaturas más bajas, el proceso térmico a $2.000^{\circ} \mathrm{K} / \mathrm{min}$.

\section{HIDRAULICIDAD DE LOS SILICATOS BICALCICOS}

Según Nurse (42) las formas $\alpha^{\prime}$ del $\mathrm{C}_{2} \mathrm{~S}$ se hidratan con dificultad y no parecen poseer propiedades hidráulicas apreciables.

Los contribuyentes minoritarios del clinker que pueden estabilizar las diferentes formas del $\mathrm{C}_{2} \mathrm{~S}$ pueden afectar la relación entre las caracteristicas hidráulicas de las diferentes formas de la belita y sus propiedades (34).

En los casos en que esto ocurre la actividad hidráulica parece depender más de la cantidad y tipo de agente estabilizador que del polimorfo obtenido.

Ono, Kawamura y Soda (44) han estudiado la belita del clinker comercial comprobando que ésta constaba de las formas $\beta$ y $\alpha$, estabilizadas por distintas proporciones de $\mathrm{Na}, \mathrm{Fe}$ y $\mathrm{Al}$. Estos autores encontraron que la forma $\alpha$ era 3 veces más reactiva que la forma $\beta$.

Suzuki (39) ha observado que la fase $\alpha$ estabilizada con K-Fe desarrollaba una resistencia mecánica más alta que las formas $\alpha^{\prime}$ y $\beta$ estabilizadas de la misma forma. Cuando utilizaba $\mathrm{Na}-\mathrm{Fe}$ como estabilizadores de la forma la resistencia mecánica disminuia. 
Jelenic, Bezkgak y Bujan (46) han estudiado la hidratación de las formas $\alpha$ ' y $\beta$ del $\mathrm{C}_{2} \mathrm{~S}$ estabilizadas con $\mathrm{B}_{2} \mathrm{O}_{3}$.

El grado de hidratación de las pastas fue determinado por difracción de rayos X. Los autores observaron que la forma $\alpha^{\prime}$ presentaba una velocidad de hidratación más alta que la forma $\beta$, la cual se traducía en una mayor resistencia a la compresión de la forma $\alpha^{\prime}$ que de la $\beta$.

Gutt, Fric y Osborne (47) afirman que la hidraulicidad del $\alpha$ '- $\mathrm{C}_{2} \mathrm{~S}$ estabilizado por 3,6 \% en peso de $\mathrm{K}_{2} \mathrm{O}$ es mayor que la del $\mathrm{B}-\mathrm{C}_{2} \mathrm{~S}$ estabilizado por un $2,1 \%$ de $\mathrm{K}_{2} \mathrm{O}$.

Welche y Gutt (7) han mostrado que la forma $\alpha$ del $\mathrm{C}_{2} \mathrm{~S}$ estabilizada con fosfato no es hidráulica, la forma $\alpha$ ' es muy débilmente hidráulica mientras que la forma $\mathrm{B}$ tiene propiedades hidráulicas relativamente altas.

Ono, Hidaka y Shirasaka (45) han comprobado por microscopía y difracción de rayos $\mathrm{X}$ que las formas $\alpha$ y $\alpha^{\prime}$ del silicato bicálcico está presente en clínkeres que contienen $\mathrm{Na}_{2} \mathrm{O}$ o $\mathrm{K}_{2} \mathrm{O}$, que la resistencia a la compresión de estos cementos que tienen las fases $\alpha$ y $\alpha^{\prime}$ son muy elevadas. Asimismo han comprobado que las resistencias de ambas fases es muy semejante y que la resistencia de la fase $\alpha^{\prime}$ estabilizada con $\mathrm{K}_{2} \mathrm{O}$ y $\mathrm{MgO}$ es más alta que la estabilizada por $\mathrm{K}_{2} \mathrm{O}$ solamente. Por el contrario, según estos autores, el efecto del $\mathrm{MgO}$ sobre la resistencia de la fase $\alpha$ es negativo.

Los resultados parecen indicar que la hidraulicidad de los distintos polimorfos del $\mathrm{C}_{2} \mathrm{~S}$ depende más del tipo y de la cantidad de agente estabilizador utilizado que del polimorfo en si y esto podría explicarse suponiendo que la hidraulicidad es función de la presencia de huecos o imperfecciones en la red cristalina. Cuando el empaquetamiento de átomos es más denso (coordinación alta de los iones $\mathrm{Ca}^{2+}$ ) la reacción de hidratación es más difícil. Por tanto aquellos elementos que al sustituir al $\mathrm{Ca}$ o al $\mathrm{Si}$ produzcan distorsiones en la red cristalina de manera que den lugar a huecos que faciliten el acceso de las moléculas de $\mathrm{H}_{2} \mathrm{O}$ mejorarán la reacción de hidratación y por tanto la hidraulicidad de los distintos polimorfos.

\section{B I B L I O G R A F I A}

(1) F. TROJAR: "The present state of Knowledge of the phase composition of portland cement clinker", Zement-KalkGips, 5, 207-215 (1966).

(2) J. W. JEFFERY: "The tricalcium silicate phase". London 1952. pp. 30-48.

(3) M. BIGARE, A. GUINIER, CH. MAZIERES, M. REGOURD, N. YANNAQUIS, W. EYSEL TH. HOLM and E. WOERMANN: "Polymorphism of Tricalcium Silicate and its Solid Solutions", J. Am. Ceram. Soc. Vol. 50 (11), 609-619 (1967).

(4) H. LAFUMA: "French basic research in the cement industry". Rev. des Materiaux de Construc. 603, 545-550 (1965) C.E.R.I.L.H. Technical Note n. ${ }^{\circ} 18$.

(5) R. W. NURSE, H. G. MIDGLEY, W. GUTT and V. E. FLETCHER: "Effect of Polymorphism of tricalcium silicate on its reactivity" Symposium on Structure of Portland Cement Paste and Concrete. Special Report 90, pp. 258-262 (1966).

(6) YN. M. BUTT and V. V. TIMASHEV: "Dependence of the binding properties of the clinker minerals on their burning temperature and crystal structure" Tsement $272,17-22$ (1961).

(7) J. H. WELCH and W. GUTT: "The effect of minor components on the hidraulicity of the calcium silicates". IV ISCC. Washington 1960, Vol. I, pp. 59-68.

(8) F. G. MIDGLEY: "Indexing of powder X-Ray diffraction data for dicalcium silicate". Trans. Brit. Ceram. Soc. 70, n. ${ }^{\circ} 2,61-63(1971)$.

(9) BELIANKIN, D. S. y LAPIN, V. V. DOKLADY: Akademii Nauk S.S.S.R. 1946 Vol. 51 p. 70 p. 
(10) GREENE, K. T.: Journal of research of the National Bureau of Standards 1944 Vo. 32 p.

(11) TILLEY, C. E.: Mineralogical Magazine and Journal of the mineralogical Society 1948. Vol. 28 p. 255.

(12) UDAGAWA: Studies on the Dusting of $\mathrm{Ca}_{2} \mathrm{SiO}_{4}$ the cristal structure of $\alpha \mathrm{L} \mathrm{Ca}_{2} \mathrm{SiO}_{4}$. p. 35-37.

(13) GUTT, W. and OSBORNE, G. J.: "The effect of potassium on the hydraulicity of dicalcium silicate. Cement Technology. 1970. 121-125.

(14) TAYLOR, W. C.: The system $2 \mathrm{CaO} \cdot \mathrm{SiO}_{2}-\mathrm{K}_{2} \mathrm{O} \cdot \mathrm{CaO} \cdot \mathrm{SiO}_{2}$ and other phase-equilibrium studies involvings potash. Journal of Research National Bureau of Standards. 1947. Vol. 27, p. 311.

(15) SMITH: Majundar and ordway. Journal of the American Ceramic Society. 1961. Vol. 44. n.o 8. 405-441. mistry of cement. 1980. Paris. powder X-Ray diffraction of 2-CaO.SiO ${ }_{2}$ ”. J. Ceram Assoc. Japan 71, n.o 1, 9-12 (1963).

(19) M. REGOURD, M. BIGARE, J. FOREST and A. GUINIER: "Synthesis and crystallographic investigation of some belites”. Supplementary paper I, 10, V-ISCC Tokyo (1968) Vol. I, 44-48 (1969).

(16) MATKOWIC, B.: Reactivity of belite stabilized by $\mathrm{Ca}_{5}\left(\mathrm{PO}_{4}\right)_{3} \mathrm{OH}$. The VII International Symposium on the chemistry of cement. 1980. Paris.

(17) TRÖMEL G. and MÖLLER, H.: Zement-Kalk-Gips, n.o 8, 235, 1952.

(18) G. YAMAGUCHI, Y. ONO, S. KAWAMURA and Y. SODA: "Differentialthermal analysis and high temperature powder X-Ray diffraction of 2-CaO.SiO ${ }_{2}$ ". J. Ceram Assoc. Japan 71, n. ${ }^{\circ}$ 1, 9-12 (1963).

(19) M. REGOURD, M. BIGARE, J. FOREST and A. GUINIER: "Synthesis and crystallographic investigation of some belites". Supplementary paper I, 10, V-ISCC Tokyo (1968) Vol. I, 44-48 (1969).

(20) N. YANNAQUIS: “X-Ray studies of silicates in clinker”. Rev. des Matériaux de Construct. 480, $213-228$ (1955) C.E.R.I.L.H. Technical Paper n.॰ 74.

(21) MIDGLEY: The VI International Congress on the chemistry of cement, section I, I-I, I-2 suplementary paper Moscow 1974.

(22) POWDER: Diffraction file, Inorganic phases. J.C.P.D.S. 1982.

(23) K. NIESEL and P. THORMAN: "The stability fields of dicalcium silicate modifications". Tonind. Ztg. 91 (a) 362-369 (1967).

(24) D. F. SMITH, A. J. MAJUMDAR y F. OROWAY: "The crystal structure of dicalcium silicate". Acta Cryst. 18, 787-795 (1965).

(25) C. M. MIDGLEY: "Crystal Structure of B-dicalcium silicate". Acta Cryst. 5, part. 3, 307-312 (1952).

(26) K. SUZUKI and G. YAMAGUCHI: "A structural study on $\alpha^{\prime}-\mathrm{Ca}_{2} \mathrm{SiO}_{4}$ ". Supplementary Paper I-92, V-ISCC Tokyo (1965) Vol. I 67-72 (1969).

(27) M. A. BREDIG: "Polymorphism of calcium orthosilicate". I. Am. Ceram. Soc. 33, 6, 188-192 (1950).

(28) G. YAMAGUCHI, Y. ONO, S. KAWAMURA e Y. SODA: "Synthesis of the varios modifications of $\mathrm{Ca}_{2}$.

(29) M. REGORD, A. GUINIER: "The crystal chemistry of the constituents of portland cement clinker" VI-ISCC Moscow 1974.

(30) N. YANNAQUIS, A. GUINIER: "Polymorphic B- transition of calcium orthosilicate" Boll. Soc. Franc. Mineral et Crist. 82, 126-136 (1959).

(31) A. A. PASNOCHENKO: Supp. paper Section I (I-4) VI ISCC. Moscow 1974.

(32) D. ROY: "Studies in the system $\mathrm{CaO}-\mathrm{Al}_{2} \mathrm{O}_{3}-\mathrm{SiO}_{2}-\mathrm{H}_{2}$. III New data on the polymorphism of $\mathrm{Ca}_{2} \mathrm{SiO}_{4}$ and its stability in the system $\mathrm{CaO}_{-} \mathrm{SiO}_{2}-\mathrm{H}_{2} \mathrm{O}$ ". J. Am. Ceram. Soc. 41 n.o 8, 293-299 (1958).

(33) V. I. KORUEEV, E. B. BYGALINC: "Thermal stabilization of $\mathrm{B}-2 \mathrm{CaO} \cdot \mathrm{SiO}_{2}$ ". V - ISCC Supplementary paper I-95.

(34) I. M. PRITTS y K. E. DAUGHERTY: "The effect of stabilizing agents on the hydration rate of B-C ${ }_{2} \mathrm{~S}$ ". Cement and Concrete Res. 6 783-796 (1976).

(35) M. GAWLICKI: W. NOCUM - Wezelik - 1-161 tomo II 7 Congress International a chemistry del ciments. Paris 1980.

(36) A. E. SHEIKIN, S. A. SLOBODCHIKOWA: "The hydraulic activity of belite in relation to the contidions of its formation and the type of stabilizer". Naudin. Soobslich - Vses. Nauch. Issled. Inst. Tsementa, 43 12, 8-13 (1961).

(37) K. SUZUKI. M. TSUJITA: "Influence of the minute quantity of impurites on the transformation of dicalcium silicates”. J. Ceram. Assoc. Japan 71. 
(38) E. THILO Y H. FUNK: "Effect of small amounts of alkalis on the B-inversion of $\mathrm{C}_{2} \mathrm{~S}$ ". Z. Anorg. Allgem. Chem. $2731-2,28-40$ (1953).

(39) K. SUZUKI: "Hydration and Strength of $-\alpha$ '-, y B-dicalcium silicates stabilized with Na-Al, K-Al, Na-Fe y K-Fe". VII ICCC Paris (1980) II-17.

(40) S. N. GHOSH: "Synthesis of dicalcium silicate and tricalcium silicate phases". VII ICCC Paris (1980) I-12.

(41) S. UDAGAWA, M. URABE: "Studies on the dusting of $\mathrm{Ca}_{2} \mathrm{SiO}_{4}$; the crystal structure of $\alpha$ '- $\mathrm{Ca}_{2} \mathrm{SiO}_{4}$ " Semento Gijyutsu Neupo 32, 31-33 (1978).

(42) R. W. NURSE: "The dicalcium silicate phase" III-ISCC. London 56-77 (1952).

(43) J. STARK, A. MULLER, R. SCHARADER and K. RÜMPER, WEIMAR: Zement-Kalk-Gips, n.o 9 (476-481) 1981.

(44) Y. ONO, S. KAWAMURA e Y. SODA: "Microscopic observations of alite and belite and hydraulic strength of cement" V ISCC Tokyo (1968) Supplementary paper I-79.

(45) Y. ONO, T. HIDAKA, M. SHIRASAKA: "On the influence of $\mathrm{Na}_{2} \mathrm{O}, \mathrm{K}_{2} \mathrm{O}$ and $\mathrm{MgO}$ on the development of strength of portland cement mortar". Review of the General Meeting. Tokio (1968) 10 p. 61-65.

(46) I. JELENIC, A. BEZJAK. M. BUJARE: "Hydration of $\mathrm{B}_{2} \mathrm{O}_{3}$ - stabilized and the modifications of dicalcium silicate". Cement and Concrete research 8, 173-180 (1978).

(47) W. GUTT, M. P. FRIC and G. J. OSBORNE: "The effect of potassium on the hydraulicity of dicalcium silicate". Cement Technology July/August 1970 p. 121-125.

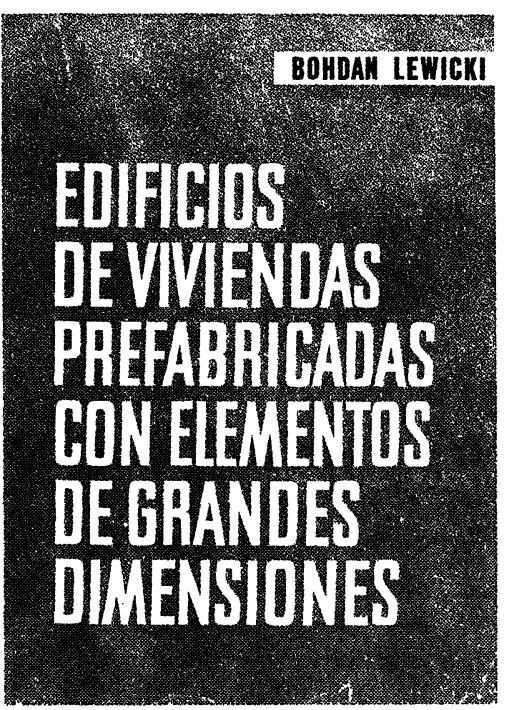

\section{Bohdan Lewicki}

Este libro trata de los problemas relativos a la construcción de los edificios de viviendas o publicos realizados con elementos prefa bricados de grandes dimenion. estudiado los problemas de arriostramiento, asi como los que plantea la resistencia de los elementos y de la estructura; se han examinado las cuestiones de orden higroter mico, acústico y de resistencia al fuego también se ha profundizado en el estudio de la estanquidad de los muros exteriores y de las juntas.

La obra incluye numerosas ilustraciones que dan detalles de diversas soluciones, asi como ejemplos de cálculo, tablas de valores numéricos, diagramas y ábacos.

Un volumen encuadernado en tela, de $24 \times 17 \mathrm{~cm}$, compuesto de 616 págs.

Precios: 2.500 ptas.; \$USA 36.00
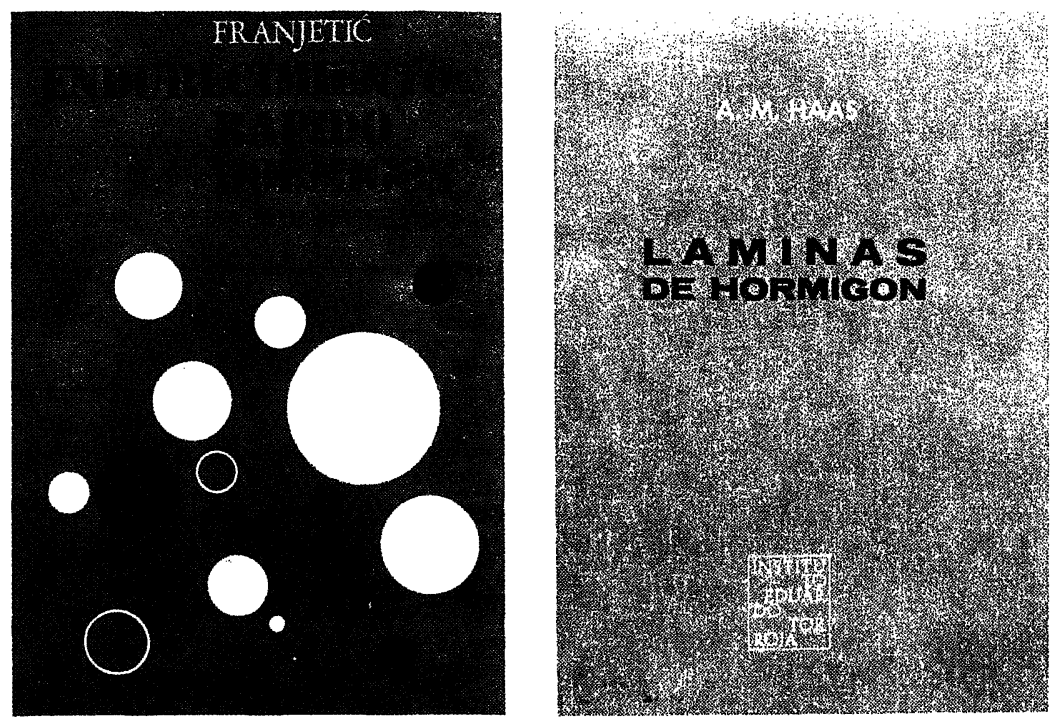

\section{Zorislav Franjetic}

En la obra de Franjetic se expone de una forma minuciosa, ordenada y sistemática, todo un cuerpo de doctrina que reúne el conocimiento actual sobre el endurecimiento rápido del hormigón. Parte el autor de los rápido del hormigón. Parte el autor de los principios básicos y llega a las últimas con-
secuencias y realidades técnicas y economicas.

Es una obra de consulta, tanto para el investigador sobre la materia, como para el proyectista y el realizador y montador de proyectista y el realizador y montador de
plantas e instalaciones y equipos de curado plantas e instalaciones y
y endurecimiento rápido.

Un volumen encuadernado en cartóné, de $17 \times 24,5 \mathrm{~cm}$, compuesto de 385 págs. 110 figuras y 10 tablas.

Precios: 2.500 ptas.; \$USA 36.00 .

\section{A. M. Haas}

Al escribir este libro el autor intentó poner a disposición de los estudiantes y de los ingenieros unos conocimientos prácticos, adecuados para servir de guia en el diseño y construcción de láminas delgadas de hormigón.

El autor está convencido de que el éxito en el diseño de una lámina exige, por parte de proyectista, un examen de las tres fases por

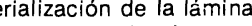
el diseño, el analisis estructural y la construcción de la estructura.

Un volumen encuadernado en tela, de $17 \times 24,5 \mathrm{~cm}$, compuesto de 420 págs , 141 figuras, 22 fotografias y 6 tablas.

Precios: 2.500 ptas.; SUSA 36.00 